\title{
Un estudio empírico sobre las actitudes ambientales
}

\author{
Juan Ignacio Aragonés y María Amérigo \\ Universidad Complutense
}

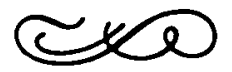

\begin{abstract}
Resumen
En esta investigación se estudia la validez de una versión castellana de la escala «Environmental Concem Scale» (ECS) llevada a cabo con 716 estudiantes. Las puntuaciones obtenidas por los sujetos proambientalistas y no proambientalistas resultan significativamente diferentes. Asimismo, se estudian las relaciones entre las actitudes bacia la preocupación por el medio ambiente, la energía nuclear y la ideología politica, mostrándose una relación entre ellas de diferente intensidad. Mientras la energía nuclear está fuertemente vinculada a la ideologia política, no sucede en tal medida entre ésta y las actitudes conservacionistas, ya que tanto los de derechas como los de izquierdas participan de la preocupación por el medio ambiente, aunque no en la misma medida.
\end{abstract}

Palabras clave: escala de preocupación ambiental, actitudes bacia el medio ambiente, energia nuclear, ideologia política.

\section{An empirical study about environmental attitudes Abstract}

This research focuses on the validity of a spanish version of the Environmental Concern Scale (ECS) carried out with 716 students. The scores obtained by pro-environmental and non pro-environmental subjects are significantly different. The relationship between attitudes towards environmental concern, nuclear power and political ideology are also studied, showing relationships between them of varying strength. Attitudes towards nuclear power are strongly linked to political ideology, whereas the link between the latter and conservationist attitudes is not so great, since right-wing, as well as left-wing subjects share in concern for the environment, although not to the same extent.

Key words: Environmental concern scale, environmental attitude, nuclear power, political ideology.

Dirección del autor: Dpto. de Psicología Social, Facultad de Psicología. Universidad Complutense. 28023 Madrid. 


\section{INTRODUCCION}

El interés por el estudio de las conductas proambientales surge en la Psicología fuertemente vinculado a acontecimientos históricos tales como la crisis energética que tiene lugar a lo largo de la década de los setenta. Entre aquellos que favorecen el estudio del comportamiento relacionado con la conservación de los recursos, pueden destacarse la subida de los precios del petróleo y la guerra entre Irán e Irak; estos dos hechos pueden ser considerados como desencadenantes de otra serie de sucesos estrechamente vinculados.

Dos enfoques claramente diferenciados dentro de la Psicología responden al interés social de estudiar la preocupación por el ambiente y sus consecuencias. Uno, vinculado a las técnicas de modificación de conducta desde un enfoque conductista, y otro, desde la perspectiva actitudinal. El primero de ellos ha sido objeto de numerosa investigación empírica como puede verse en las revisiones realizadas por Aragonés (1985), Blas y Aragonés (1986) y Geller (1987). $\mathrm{El}$ segundo tratamiento del problema medioambiental no tuvo tanta implantación, como en un principio podría esperarse, debido a que en el mismo momento en que tiene lugar la crisis energética en el mundo surge la fuerte polémica entre los psicólogos sociales a propósito de si la actitud predice o no la conducta. Las alternativas a este dilema que emergen con posterioridad, como el modelo de Fishbein y Ajzen (1975), permiten un análisis de la realidad más válido sobre el funcionamiento de la actitud, que el mero planteamiento mecanicista; de aquí que el concepto actitud emerja de nuevo con más fuerza, si cabe, de la que tuvo en la década anterior.

Las investigaciones sistemáticas sobre las actitudes proambientales comienzan a tomar interés en la Psicología Ambiental al comienzo de la década de los setenta, siendo en la actualidad un campo claramente definido en la materia, como han puesto de manifiesto, entre otros, Darley y Gilbert (1985), Gray (1985), Milbrath (1986) y Gifford (1987).

En un breve recorrido histórico sobre el estudio de la medida de las actitudes proambientales hay que reconocer que Van Liere y Dunlap (1981) que en un primer momento se trabaja en las investigaciones con técnicas que permiten un tratamiento estadístico bastante pobre. En la mayoría de los casos, se utilizaba un sólo ítem para medir la tendencia de la actitud proambiental. Muy rápidamente se desarrollan estrategias multi-ítem, permitiendo de esta forma, un mayor potencial estadístico, y por consiguiente la posibilidad de estudiar relaciones más complejas entre las variables que se ponen en relación. Es en este momento cuando tienen lugar investigaciones como la Tognacci et al. (1972), Maloney y Ward (1973) y Maloney, Ward y Braucht (1975), por destacar algunas de las más consideradas en la bibliografía sobre el tema en este período. En todas ellas se trata de poner a prueba las relaciones que existen entre varios aspectos que se consideraban relacionados con la preocupación ambiental y diversas variables sociodemográficas. En estos casos se construyeron escalas de actitudes con formato tipo Likert compuestas cada una de ellas por varias subescalas.

Un intento de investigar empíricamente la compatibilidad de distintas escalas y subescalas sobre la preocupación ambiental fue llevado a cabo por Var Liere y Dunlap (1981). Como reconocen estos autores, hasta el momento se había diseñado multitud de escalas para medir la preocupación ambiental, suponiendo los investigadores que las subescalas que componían cada una de las escalas medían algún aspecto del mismo constructo. Los mencionados autores investi- 
gan empíricamente dos conceptos diferentes. En primer término, lo que denominan «sustantive issues» (temas sustantivos) incluidos en la medida, es decir las cuestiones ambientales que contiene cada escala y que se supone forman parte de las dimensiones del concepto «preocupación ambiental». La práctica habitual había sido considerar distintos temas ambientales dentro de la preocupación ambiental y diseñar una escala que los contemplara sin atender nada más que al sentido común a la hora de comprobar si esos temas forman parte del constructo que se define como preocupación ambiental. Hasta ese momento no ha habido un intento serio de realizar una comprobación empírica de si tales temas estaban bajo un concepto más amplio como el de preocupaoión ambiental.

El otro aspecto, denominado «theoretical conceptualization» (conceptualización teórica) se refiere a las bases teóricas usadas para desarrollar medidas de preocupación ambiental. Aunque este aspecto es muy interesante de estudiar, no parece que este contexto sea el más idóneo para discutirlo, ya que la opción empírica realizada en esta investigación está estrechamente vinculada a la medición de actitudes a través de la clásica escala verbal tipo Likert.

Siguiendo a Van Lier y Dunlap (1981) en su revisión precedente al trabajo empírico, ellos hacen notar la insistente aparición de la influencia de las variables sociodemográficas en la preocupación ambiental en todos los trabajos empíricos llevados a cabo sobre esta problemática. La inclusión de estas variables permite estudiar las posibilidades de incidir en el futuro en la consolidación o en el cambio de actitudes de diversos grupos de la población, según sean sus características sociodemográficas.

En el modelo que proponen estos autores se trata de plantear la cuestión de que los temas sustantivos de una escala que mide preocupación ambiental deben correlacionar altamente. Asimismo, se pone de manifiesto que las correlaciones entre las medidas de los temas sustantivos y las variables sociodemográficas deberían ser prácticamente iguales.

A pesar dél intento fallido en la comprobación empííca del modelo, los autores concluyen que en el concepto de preocupación ambiental es bastante amplio, pero los temas que mejor le representan son la «polución» y los «recursos naturales» (Van Liere y Dunlap, 1981). Por lo que se refiere a las variables sociodemográficas, sus resultados no difieren de los que en repetidas investigaciones han surgido (Tognaci et al., 1972; Weigel, 1977, Wohlwill, 1979, y Van Liere y Dunlap, 1980), reiterándose que las que están más relacionadas con la preocupación ambiental son la ideología política y el nivel de educación. En este sentido, cuanto más a la izquierda y mayor sea el nivel de educación mayor es la preocupación ambiental manifestada por los sujetos en la escala.

Un campo que ha sido estudiado en gran número de ocasiones vinculado a la preocupación ambiental es la actitud hacia la energía nuclear. Investigaciones tales como las de Hensler y Hensler (1979) y Webber (1982), entre otros, han puesto de manifiesto una correlación alta entre ambas actitudes; llegando este último autor a comprobar que las actitudes hacia la energía nuclear tienen fuertes implicaciones ideológicas y partidistas, teniendo además relaciones con la edad y el nivel de ingresos con mayor intensidad que las actitudes proambientales. A juicio de Webber, las actitudes hacia la energía nuclear es un asunto más conflictivo que las actitudes ambientales en general.

En un trabajo posterior, Levi y Holder (1986) tratan de poner a prueba entre otras cuestiones en qué medida las actitudes antinucleares son función de 


\section{6}

la preocupación ambiental en un sentido amplio. En sus conclusiones, ponen de manifiesto que aunque la energía nuclear y el ambientalismo están relacionados, son temas diferentes, estando el primero en un mismo nivel que otros temas vinculados con la conservación de la energía.

Una serie de trabajos llevados a cabo por Van der Plig y colaboradores a lo largo de la década de los ochenta, han ido poniendo de manifiesto la importancia que las actitudes hacia la energía nuclear tenían en los países occidentales, vinculadas a la percepción del riesgo. Esta percepción se construye sobre valores, actitudes y un conjunto de atributos que no tienen por qué tener su correspondencia con las de los técnicos y expertos del tema, ni con las de los políticos que toman decisiones sobre el establecimiento de una planta nuclear en un lugar determinado. Van de Plig, Van der Linden y Ester (1982) establecen dos posibilidades para estudiar el papel que juega la energía nuclear desde el punto de vista de su aceptabilidad pública: en primer término, los estudios sobre la percepción del riesgo, siguiendo la línea de Fischhoff et al. (1981); y en segundo lugar, los desarrollados por Otway y Fisbhein (1976) sobre la estructura actitudinal. Desde esta segunda perspectiva, trabajos empíricos como los de Van der Plig (1985) llevan a afirmar que la actitud hacia la energía nuclear está fuertemente situada dentro del dominio de lo político y la moral. Asimismo, se produce una actitud más favorable en las comunidades que viven cerca de una central existente que en los que viven alejados (Van der Plig, Eiser y Spreas, 1986).

En resumen, como señala Webber (1982), aunque la energía nuclear puede ser considerada como un tema ambiental, las actitudes sobre ambos conceptos sostienen fuertes diferencias. Así, es frecuente que se dé el caso de que los defensores de la energía nuclear obtengan apoyo de personas preocupadas por el medio ambiente; sin embargo, aquellos que se sitúan en contra de la energía nuclear no siempre tienen tal apoyo.

Tanto en las investigaciones llevadas a cabo sobre las actitudes ambientales como sobre la energía nuclear, se han estudiado las relaciones que éstas tenían con ciertas variables sociodemográficas; resultando en la gran mayoría de ellas la edad, el nivel de estudio y la ideología política, las variables con mayor influencia sobre las mencionadas actitudes.

Los tres campos tratados hasta el momento: actitudes proambientales, actitudes hacia la energía nuclear y variables sociodemográficas, están estrechamente relacionadas, mostrando una relación compleja, en la que es necesario trabajar con el propósito de encontrar un marco en el que se pueda dar respuesta a las relaciones que existen entre ellas. Para poder abordar esta problemática en las coordenadas socioculturales de España, es necesario comenzar por diseñar o retomar un instrumento de medida, dada la ausencia de este tipo de trabajos en estas latitudes. El uso de medidas estandarizadas tal y como señalan Van Liere y Dunlap (1981) permite facilitar las comparaciones entre estudios y ayuda a la acumulación de datos empíricos, de tal forma que se puedan generalizar los resultados. De aquí que una buena elección del instrumento sea importante por comprometer la investigación futura.

Por tanto, el primer objetivo de esta investigación es tratar de probar la validez de la escala de actitudes proambientales diseñada por Weigel y Weigel (1978) denominada Environmental Concern Scale (ECS) (Escala de Preocupación Ambiental) en una versión traducida al español y aplicada a un grupo de españoles. 
El segundo objetivo consiste en ver en qué medida existen relaciones entre los tres niveles comentados: las actitudes hacia la protección del medio ambiente y las actitudes ante la energía nuclear y la ideología política.

\section{METODO}

\section{MUESTRA}

La muestra utilizada fue de 716 estudiantes de psicología de distintos cursos, de los cuales 125 eran varones y 591 eran mujeres. El status socioeconómico que se atribuían los sujetos era medio-medio con cierta tendencia a medio-alto. Por tanto, esta variable, el nivel de educación y la edad que se encuentra comprendida en un intervalo de 19 a 29 años, hacen que la muestra pueda ser considerada homogénea en todas estas variables. Las características de la muestra permiten evaluar con mayor énfasis los resultados debidos a las variables sexo e ideología política, si cabe, ya que se pueden considerar a las tres anteriores controladas, siendo además de gran interés en la temática de que se ocupa esta investigación.

\section{INSTRUMENTOS}

Para cubrir los objetivos propuestos se diseñó un cuestionario al efecto, que consta de cuatro partes claramente diferenciadas: Escala de Preocupación Ambiental (Weigel y Weigel, 1978), un ítem para medir la tendencia actitudinal hacia la energía nuclear, una serie de cuestiones relativas a la conducta de voto e ideología política y, finalmente, un apartado dedicado a recoger las variables sociodemográficas (ver apéndice).

\section{RESULTADOS}

\section{a) Escala de Preocupación Ambiental}

La ausencia de instrumentos en castellano que midieran las actitudes hacia el medio ambiente invitó a realizar un recorrido por la literatura sobre esta temática, que aconsejara de una forma más evidente la opción por alguna de las escalas ya utilizadas. La revisión se realizó utilizando dos criterios. En primer término, y como señalan Weigel y Weigel (1978), p. 3), «que la herramienta fuera válida para medir las actitudes capaces de valorar unos sentimientos y creencias relativamente constantes de los individuos sobre la conservación del ambiente, de tal modo que tales predisposiciones permitan saber en qué medida pueden ser anticipadas las conductas proambientales». En segundo lugar, que contemplara los «temas sustantivos» de la preocupación ambiental comprobados empíricamente en la investigación de Van Liere y Dunlap (1981).

Ambos criterioś, aconsejaron tomar la conocida escala de Environmental Concern Scale (ECS) de Weigel y Weigel (1978) en lengua inglesa, dado que los temas sustantivos sobre los que versa son la conservación y contaminación del medio ambiente que son precisamente los que Van Liere y Dunlap (1981) pusieron de manifiesto como los más indicados para medir la preocupación ambiental. Asimismo, esta es una de las escalas que con mayor frecuencia ha sido utilizada en la investigación empírica, lo que facilita la comprobación de resultados en los distintos trabajos. 
TABLA 1

Saturación de los items en los 4 factores

\begin{tabular}{|c|c|c|c|c|}
\hline FACTOR & 1 & 2 & 3 & 4 \\
\hline $\begin{array}{l}\text { 1. Si pudiera daría tiempo, dinero o ambos para una or- } \\
\text { ganización como ADENA que trabaje para mejorar } \\
\text { la calidad del ambiente. }\end{array}$ & 0,774 & & & \\
\hline $\begin{array}{l}\text { 2. Estaría dispuesto a aceptar un incremento de mis gas- } \\
\text { tos a } 5.000 \text { ptas. el próximo año para promover el } \\
\text { uso prudente de los recursos naturales. }\end{array}$ & 0,562 & & & \\
\hline $\begin{array}{l}\text { 3. Estaría dispuesto a hacer sacrificios personales para } \\
\text { reducir el ritmo de la contaminación aunque los re- } \\
\text { sultados inmediatos no puedan parecer significativos. }\end{array}$ & & 0,406 & & 0,254 \\
\hline 4. La contaminación no afecta personalmente a mi vida. & & 0,318 & & \\
\hline $\begin{array}{l}\text { 5. Los beneficios de los productos de consumo moder- } \\
\text { nos son más importantes que la contaminación que } \\
\text { resulta de su producción y uso. }\end{array}$ & & 0,636 & & \\
\hline $\begin{array}{l}\text { 6. La actividad corriente de las organizaciones anticon- } \\
\text { taminación está realmente más interesada en romper } \\
\text { con la sociedad que en luchar contra la contamina- } \\
\text { ción. }\end{array}$ & & 0,281 & & \\
\hline $\begin{array}{l}\text { 7. Incluso si el transporte público fuera más eficiente } \\
\text { de lo que es, yo prefiriría llevar mi coche al trabajo. }\end{array}$ & & 0,368 & & \\
\hline $\begin{array}{l}\text { 8. Aunque hay contaminación continua de lagos, ríos } \\
\text { y aire, los procesos de purificación de la naturaleza } \\
\text { pronto los retornan a lo normal. }\end{array}$ & & & 0,559 & \\
\hline $\begin{array}{l}\text { 9. Es muy improbable que la contaminación debida a } \\
\text { la producción de energía llegue a ser excesiva, por- } \\
\text { que el gobierno tiene muy buenas inspecciones y agen- } \\
\text { cias de control. }\end{array}$ & & & 0,486 & \\
\hline $\begin{array}{l}\text { 10. La industria está haciendo los mayores esfuerzos po- } \\
\text { sibles para desarrollar tecnología anticontaminante } \\
\text { efectiva. }\end{array}$ & & & 0,431 & \\
\hline $\begin{array}{l}\text { 11. No deberíamos preocuparnos por matar demasiados } \\
\text { animales de caza porque a la larga las cosas se equili- } \\
\text { brarán. }\end{array}$ & & & 0,265 & \\
\hline $\begin{array}{l}\text { 12. En la escuela pública deberán ser impartidos cursos } \\
\text { sobre la conservación de recursos naturales. }\end{array}$ & & & & 0,655 \\
\hline $\begin{array}{l}\text { 13. Debemos prevenir la exitinción de cualquier tipo de } \\
\text { animal, incluso si ello significa sacrificar algunas co- } \\
\text { sas para nosotros mismos. }\end{array}$ & & & & 0,486 \\
\hline
\end{tabular}

La presentación de esta escala de tipo Likert de 5 puntos fue idéntica a la utilizada por sus creadores. Los 16 ítems de que consta fueron presentados, nueve de ellos de forma positiva y siete de forma negativa. Tras su traducción y adaptación al castellano fue sometida a pruebas de consistencia y validez.

La consistencia interna de la escala fue hallada a través de coeficiente $\alpha$ obteniendo un valor de 0,74 , ligeramente inferior al obtenido por Weigel y Weigel 
(1978) de 0,85 . No obstante, el valor obtenido es suficientemente elevado como para considerar la escala con una consistencia interna satisfactoria.

Con el propósito de establecer en qué medida la escala medía el constructo que se propone, es decir, las actitudes ambientales, se sometieron las respuestas a los distintos ítems que formaban dicha escala a un análisis factorial tipo MFLA. En el primer análisis emergieron 4 factores cuyas saturaciones en los ítems correspondientes aparecen en la Tabla 1 . Los ítems situados en los lugares 1, 10 y 11 de la escala (ver Apéndice 1) no alcanzaron un valor de saturación superior a 0,25 ninguno de ellos. Los cuatro factores resultantes pueden ser definidos en una primera aproximación como sigue: Esfuerzo económico (Factor 1), Contaminación (Factor 2), Control no personal (Factor 3) y Conservación (Factor 4).

Como el objetivo que se perseguía con este análisis era comprobar en qué medida aparecía un solo factor en todos los ítems de la escala, de tal forma que se pudiera establecer la validez del constructo, los resultados obtenidos fueron sometidos a un análisis factorial de segundo orden. En él emergió un solo factor, lo que permite afirmar que todos y cada uno de los ítems están implicados en un constructo específico.

La validez de la escala fue además estudiada a través de dos ítems que se habían incluido en el cuestionario a tal efecto. El primero de ellos, tomado del trabajo de Dunlap (1975), es el siguiente: «¿Cuánto interés tiene en los problemas ambientales?" y pretender medir la orientación general de los sujetos con respecto a los problemas ambientales. La respuesta se registraba en una escala de cuatro puntos desde gran interés hasta ningún interés. El segundo ítem «Necesitamos leyes fuertes para proteger el ambiente incluso aunque aumenten los precios y se reduzca el número de puestos de trabajo» fue tomado de un trabajo realizado por Webber (1982) en el que desaparece el efecto de deseabilidad social que puede contener el primero. Las respuesta se producían en una escala de siete puntos desde muy de acuerdo hasta muy en desacuerdo.

Las puntuaciones en el primer ítem fueron relacionadas con las obtenidas por los sujetos en la escala a través de un análisis de varianza y su correspondiente análisis de Scheffe, resultando una $F=84,1 ; p<0,001$, lo que permite afirmar que las puntuaciones obtenidas en la escala son significativamente diferentes entre todos los grupos correspondientes al ítem, desde gran interés hasta ningún interés en una proporción menor de 0,01 .

Como se ha comentado anteriormente, el efecto de deseabilidad social podía haber contaminado la respuesta de este ítem, y esto puede ser confirmado por el hecho de que sólo el $1,3 \%$ de los sujetos respondieron no tener ningún interés por los temas ambientales. Como alternativa, el otro ítem sitúa al sujeto entre optar por el ambientalismo o por dos de los problemas que más azotan a la economía capitalista en estos momentos: el paro y la inflación. La tasa de respuesta se distribuye en función del ítem anterior tal y como aparece en la Tabla 2.

La correlación entre ambos ítems es de 0,312 , lo que de alguna forma confirma lo que se venía comentando, ya que si se observa la Tabla 4 se puede ver que el $15,8 \%$ de los sujetos están puntuando en el ítem en alguna de sus variantes: muy y bastante en desacuerdo, mientras que en el item anterior sólo un $1,3 \%$ no tenía ningún interés por los temas ambientales. Estos resultados parecen establecer que los sujetos no son tan extremadamente ambientalistas como se podría haber concluido de las respuestas dadas al ítem primero; porque cuando se les pone ante la disyuntiva de ambientalismo frente a la inflación y al 
paro, las posiciones retroceden, y muy probablemente a posiciones más coincidentes con la realidad cotidiana, sobre todo en muestras de características similares a las utilizadas en este trabajo.

\section{TABLA 2}

Relación en porcentajes entre los items. ¿Cuánto interés tienes por los problemas ambientales? (columnas) y inecesitamos leyes fuertes de protección del ambiente aunque aumenten el paro y los precios? (filas)

\begin{tabular}{|l|r|r|r|r|r|}
\hline & \multicolumn{5}{|c|}{ I N T E R E S } \\
\hline & GRAN & BASTANTE & ALGUN & NINGUN & TOTAL \\
\hline Muy desacuerdo & 4,3 & 4,3 & 4,7 & 33,3 & 4,8 \\
Bastante desacuerdo & 4,3 & 11,1 & 14,6 & 33,3 & 11,0 \\
Algo desacuerdo & 14,4 & 24,6 & 38,5 & 11,1 & 26,2 \\
Indiferente & 1,4 & 1,7 & 4,7 & 11,1 & 2,6 \\
Algo de acuerdo & 30,2 & 39,1 & 27,1 & 11,1 & 33,6 \\
Bastante de acuerdo & 25,9 & 16,6 & 8,3 & 0,0 & 15,9 \\
Muy de acuerdo & 19,4 & 2,6 & 2,1 & 0,0 & 5,8 \\
\hline
\end{tabular}

Como una segunda prueba de validez, con pretensiones de ser mejor que la anterior, se relacionaron las puntuaciones obtenidas en la escala con las respuestas dadas en este último ítem. Estas fueron agrupadas de tal forma que resultaron cinco grupos: muy y bastante de acuerdo, algo de acuerdo, indiferente, algo en desacuerdo y muy y bastante en desacuerdo.

Como se hizo en el caso anterior, se realizó un análisis de varianza, y los resultados permiten afirmar que las puntuaciones obtenidas en la escala están en relación con las respuestas dadas al ítem $(\mathrm{F}=26,87 ; \mathrm{p}<0,001)$. Un análisis de Scheffe permitió establecer en qué medida las diferencias se producen en los cinco grupos establecidos a partir del ítem, desde muy y bastante de acuerdo hasta muy y bastante en desacuerdo. Los resultados aparecen en la Tabla 3 y merecen los siguientes comentarios: los sujetos que muestran en el ítem conductas proambientales; es decir, los muy, bastante y algo de acuerdo con la creación de leyes fuertes que protejan el ambiente, puntúan en la escala no sólo significativamente diferente al resto de los grupos, sino que incluso presentan diferencias significativas entre ellos mismos. Por el contrario, los que dan respuestas no ambientalistas en el ítem muy, bastante y algo en desacuerdo, no son discriminados entre ellos por la escala de forma significativa.

En resumen, la escala discrimina entre los proambientalistas y no proambientalistas, así como entre los primeros, según el ítem que se viene comentando. No obstante, debe continuarse con otras pruebas más potentes que confirmen su validez utilizando criterios externos de validación. Para ello deberán utilizarse sujetos con probadas conductas ambientalistas pertenecientes a organizaciones de protección del medio ambiente $y$, sujetos con conductas antiambientalistas, tales como explotadores con afán sólo de lucro en la obtención de recursos naturales. Esto permitiría confirmar si la esca no sólo discrimina entre ambientalistas y antiambientalistas sino también, entre los primeros, como parece desprenderse de los resultados obtenidos. De ser así, subyacerían actitudes 


\section{1}

TABLA 3

Nivel de significación tras la prueba de Scheffe entre los cinco grupos correspondientes al item: «Necesitamos leyes fuertes para proteger el ambiente, incluso aunque aumenten los precios y se reduzca el número de puestos de trabajo» $(1=p<0,01)$ con la escala ECS

\begin{tabular}{|l|c|c|c|c|c|}
\hline & $\begin{array}{c}\text { MUY, BAST. } \\
\text { DESACUER. }\end{array}$ & $\begin{array}{c}\text { ALGO } \\
\text { DESACUER. }\end{array}$ & INDIFEREN. & $\begin{array}{c}\text { ALGO } \\
\text { ACUERDO }\end{array}$ & $\begin{array}{c}\text { MUY, BAST. } \\
\text { ACUERDO }\end{array}$ \\
\hline $\begin{array}{l}\text { MUY, BAST. } \\
\text { DESACUER. }\end{array}$ & & & & & \\
\hline $\begin{array}{l}\text { ALGO } \\
\text { DESACUER. }\end{array}$ & & 1 & & & \\
\hline INDIFER. & 1 & 1 & 1 & & \\
\hline $\begin{array}{l}\text { ALGO } \\
\text { ACUERDO }\end{array}$ & 1 & 1 & 1 & 1 & \\
\hline $\begin{array}{l}\text { MUY, BAST. } \\
\text { ACUERDO }\end{array}$ & 1 & 1 & & & \\
\hline
\end{tabular}

cualitativamente distintas entre aquellos que realizan conductas proambientales y los que manifiestamente actúan en contra de la calidad ambiental. De esta forma, la escala mediría sólo el aspecto positivo de la relación del hombre con el medio ambiente.

Con referencia a las puntuaciones obtenidas por los sujetos de la muestra . en la escala, merecen destacarse los siguientes resultados: las puntuaciones de los sujetos son superiores a su valor medio 48 , en todos los casos excepto en dos de ellos y la puntuación media obtenida es de 64,4 y una desviación típica de 6,6. Estos resultados muestran la fuerte preocupación por los problemas ambientales que tienen los sujetos encuestados. A la luz de la bibliografía sobre el tema era de esperar esta favorable actitud proambiental de los sujetos, dadas sus características de status socioeconómico y nivel de educación.

Con el propósito de ver en qué medida las puntuaciones obtenidas en la escala estaban relacionadas con la edad y el sexo, se realizó un análisis de varianza cuyos resultados muestran la inexistencia de relación entre ambas variables las actitudes ambientales. (Sexo, $F=0,04, p=0,848$; Edad, $F=1,86$, $\mathrm{p}=0,134)$.

\section{b) Actitudes hacia la energia nuclear}

Con el ítem utilizado al efecto en el cuestionario, no se pretendía medir la actitud hacia las centrales de energía nuclear, sino obtener la tendencia sobre esta problemática para ver su relación con otras variables como la actitud hacia la conservación del ambiente y la ideología política. El ítem fue tomado del trabajo de Levi y Holder (1986) en el que se estudian las relaciones entre las actitudes hacia la energía nuclear y las actitudes ambientales medidas a través de la ECS de Weigel y Weigel (1978). Fue presentado en los siguientes términos:

¿Cuál de las siguientes frases está más próxima a su opinión?

- Todas la centrales nucleares de España deberían cerrarse.

- Deberían permitirse que continuaran funcionando todas aquellas centrales que actualmente funcionan, pero no deberían construirse nuevas centrales.

- Deberían seguirse construyendo nuevas plantas nucleares. 
La Tabla 4 correspondiente a la distribución de las respuestas de los sujetos en función de la edad y el sexo, permite afirmar que la mayoría de la muestra, el $50,4 \%$, desea que se paren los programas de instalación de nuevas centrales en España y un 39,3\% piensa que deberían incluso pararse las que están en funcionamiento. La suma de ambos porcentajes está en línea con el trabajo de Levi y Holder (1986), también realizado con estudiantes. Estos autores obtienen unos porcentajes del $56 \%$ y $25 \%$ respectivamente, lo que sugiere que los estudiantes de la muestra aquí considerada mantienen una posición ligeramente más radical contra la energía nuclear que los estudiantes americanos entrevistados.

TABLA 4

Porcentajes de las respuestas sobre actitudes a la energia nuclear según la edad y sexo

\begin{tabular}{|c|c|c|c|c|}
\hline \multicolumn{5}{|c|}{ E D A D } \\
\hline & $19-20$ & $21-22$ & $23-24$ & $N>24$ \\
\hline $\begin{array}{l}\text { No centrales nucleares } \\
\text { No más centrales nucleares } \\
\text { Sí más centrales nucleares }\end{array}$ & $\begin{array}{r}35,1 \\
55,5 \\
9,4 \\
\end{array}$ & $\begin{array}{l}39,8 \\
48,4 \\
11,9\end{array}$ & $\begin{array}{l}37,4 \\
51,0 \\
11,6\end{array}$ & $\begin{array}{r}50,0 \\
44,6 \\
5,4\end{array}$ \\
\hline \multicolumn{5}{|c|}{$\mathrm{S} \mathrm{EXO}$} \\
\hline & VARON & & & TOTAL \\
\hline $\begin{array}{l}\text { No centrales nucleares } \\
\text { No más centrales nucleares } \\
\text { Sí más centrales nucleares }\end{array}$ & $\begin{array}{l}43,8 \\
37,2 \\
19,0\end{array}$ & & & $\begin{array}{l}39,4 \\
50,4 \\
10,2\end{array}$ \\
\hline
\end{tabular}

Cuando se trata de examinar la influencia de las variables sociodemográficas edad y sexo sobre las respuestas dadas al ítem, se observa que la edad no $\mathrm{da}$ lugar a diferencias significativas $\left(\chi^{2}=8,603 ; \mathrm{p}=0,1971\right)$. Sin embargo, el sexo sí ofrece diferencias $\left(\chi^{2}=17,094 ; p<0,001\right)$, observándose en las mujeres una mayor tendencia al rechazo de la energía nuclear.

\section{c) Ideología política}

Esta variable fue operativizada en el cuestionario a través de dos ítems. El primero de ellos se refería a la conducta de voto de los sujetos en elecciones generales correspondientes a 1986; y el segundo, a la ideología que ellos se autoatribuían en un abanico que abarca desde la extrema derecha hasta la extrema izquierda.

Las razones de estos dos ítems obedecen a la estrategia ya clásica desarrollada por Dunlap (1975) en este tipo de estudios. Este autor operativizó la variable ideología política atendiendo a la conducta de voto y a la autoatribución ideológica a lo largo de una escala. Entre los resultados que aporta en su trabajo, destaca la mayor capacidad de explicación del segundo criterio frente al primero; sin bien, debe hacerse notar que en EE.UU. los dos partidos, Republicano y 
Demócrata, abarcan amplios espectros ideológicos. En el caso presente se optó por la variable autoatribución de la ideología política, ya que el voto puede ser en muchos casos fruto de una situación coyuntural, mientras que la ideología parece más estable. En esta línea, Buttel y Flinn (1978) confirman que la identificación con el partido es menos importante que la ideología para explicar las actitudes ambientales.

La distribución de las respuestas con respecto a la conducta de voto, atendiendo a las variables sociodemográficas de edad y sexo, aparece en la Tabla 5 . Ninguna de ellas parece tener influencias significativas sobre la emisión del voto. Un análisis $\chi^{2}$, arroja los siguientes resultados $\chi^{2}=24,651 ; \mathrm{p}=0,0548$ para la edad y $\chi^{2}=8,294 ; \mathrm{p}=0,1408$ para el sexo.

TABLA 5

Porcentajes de las respuestas sobre el voto emitido según la edad y el sexo

\begin{tabular}{|c|c|c|c|c|}
\hline \multicolumn{5}{|c|}{ E D A D } \\
\hline & $19-20$ & 21.22 & $23-24$ & $N>24$ \\
\hline $\begin{array}{l}\text { CP.PR } \\
\text { CDS } \\
\text { PSOE } \\
\text { IU } \\
\text { OTRO } \\
\text { NO VOTO }\end{array}$ & $\begin{array}{r}19,7 \\
14,9 \\
21,3 \\
11,2 \\
8,5 \\
24,5\end{array}$ & $\begin{array}{r}24,4 \\
9,5 \\
22,7 \\
12,8 \\
8,3 \\
22,3 \\
\end{array}$ & $\begin{array}{r}18,1 \\
7,6 \\
34,0 \\
8,3 \\
7,6 \\
24,3 \\
\end{array}$ & $\begin{array}{r}9,8 \\
8,7 \\
34,8 \\
15,2 \\
9,8 \\
21,7 \\
\end{array}$ \\
\hline \multicolumn{5}{|c|}{ S E X O } \\
\hline & VARON & & & TOTAL \\
\hline $\begin{array}{l}\text { CP-PR } \\
\text { CDS } \\
\text { PSOE } \\
\text { IU } \\
\text { OTRO } \\
\text { NO VOTO }\end{array}$ & $\begin{array}{l}12,6 \\
10,1 \\
24,4 \\
13,4 \\
12,6 \\
26,9\end{array}$ & & & $\begin{array}{r}19,4 \\
10,7 \\
26,6 \\
11,8 \\
8,2 \\
23,2\end{array}$ \\
\hline
\end{tabular}

Los resultados obtenidos sobre la ideología política que se atribuyen a sí mismos los sujetos, atendiendo a las variables de clasificación edad y sexo, aparece reflejada en la Tabla 6 . Obsérvese que se han unido las respuestas de extrema derecha y derecha, así como las de izquierda y extrema izquierda, dado el bajo número de sujetos que puntuaron en las opciones extremas $0,4 \%$ y $1,3 \%$, respectivamente.

Sometidas las frecuencias a un análisis estadístico $\chi^{2}$ se obtuvieron los siguientes resultados: $\chi^{2}=15,682: p=0,2062$ para la variable edad y $\chi^{2}=13,213$; $\mathrm{p}=0,0103$ para el sexo. Esto permite afirmar que las mujeres se perciben a sí mismas más de derechas que los hombres, aunque ambos no se diferencien en la conducta de voto propiamente dicha.

Siguiendo el trabajo de Dunlap (1975) con el propósito de utilizar un solo valor de la variable, se calculó la correlación entre aquellos que habían votado 


\section{4}

TABLA 6

Porcentaje de las respuestas sobre ideología política autoatribuida según la edad y sexo

\begin{tabular}{|c|c|c|c|c|}
\hline \multicolumn{5}{|c|}{ E D A D } \\
\hline & $19-20$ & $21-22$ & $23-24$ & $N>24$ \\
\hline $\begin{array}{l}\text { IZDA. } \\
\text { C-IZDA. } \\
\text { CENTRO } \\
\text { C-DCHA. } \\
\text { DCHA. }\end{array}$ & $\begin{array}{r}39,8 \\
25,3 \\
19,2 \\
18,1 \\
6,6\end{array}$ & $\begin{array}{r}37,2 \\
26,6 \\
7,0 \\
16,7 \\
13,0\end{array}$ & $\begin{array}{r}68,6 \\
20,7 \\
5,0 \\
17,1 \\
8,6 \\
\end{array}$ & $\begin{array}{r}51,8 \\
21,7 \\
8,4 \\
12,0 \\
6,0 \\
\end{array}$ \\
\hline \multicolumn{5}{|c|}{$S E X O$} \\
\hline & VARON & & & TOTAL \\
\hline $\begin{array}{l}\text { IZDA. } \\
\text { C-IZDA. } \\
\text { CENTRO } \\
\text { C-DCHA. } \\
\text { DCHA. }\end{array}$ & $\begin{array}{r}56,3 \\
18,8 \\
8,9 \\
19,7 \\
5,4\end{array}$ & & & $\begin{array}{r}42,4 \\
24,4 \\
7,6 \\
16,6 \\
9,1\end{array}$ \\
\hline
\end{tabular}

alguna de las opciones políticas más representativas y la ideología que los sujetos se habían autoatribuido. El valor obtenido fue de 0,73 (ver Tabla 7). Este es satisfactorio, sin embargo, conviene comentar algunos aspectos a la luz de los resultados. En primer término, se observan ciertos solapamientos, así el $40 \%$ de los que votaron CDS puntúan en centro-izquierda, al igual que los que votaron PSOE, un $40 \%$ de éstos se sitúan también en la misma posición. Prácticamente la totalidad de IU $(92 \%)$ se considera de izquierda y el $57,1 \%$ de los que votaron al PSOE. Estos y otros solapamientos aconsejan recurrir a realizar los análisis con la variable autoatribución de la ideología política, máxime si se tiene en cuenta que el número de sujetos aumentará dado que se recogieron los que se abstuvieron de votar y aquellos que votaron a otras opciones políticas no consideradas en el cuestionario.

TABLA 7

Relación en porcentajes entre el voto emitido e ideología autoatribuida

\begin{tabular}{|l|r|r|r|r|r|}
\hline & CP & CDS & PSOE & \multicolumn{1}{|c|}{ IU } & TOTAL \\
\hline IZDA. & 0,0 & 5,6 & 57,1 & 92,4 & 38,9 \\
C-IZDA. & 4,8 & 40,3 & 40,0 & 6,3 & 24,2 \\
CENTRO & 4,0 & 33,3 & 1,8 & 0,0 & 7,2 \\
C-DCHA. & 54,0 & 19,4 & 1,2 & 0,0 & 18,8 \\
DCHA. & 37,3 & 1,4 & 0,0 & 1,3 & 11,0 \\
\hline
\end{tabular}

Tras dar cuenta del primer objetivo y de los resultados descriptivos de la muestra, de forma independiente en cada una de las variables criterio conside- 
radas: actitudes proambientales, actitudes hacia la energía nueclar e ideología política, puede pasarse a comentar en qué medida se ha cubierto el segundo objetivo, referido a las relaciones entre las tres variables.

\section{A) Ambientalismo e ideología política}

Como se acordó en el apartado anterior, la relación entre ambas variables se estudió en función de las puntuaciones obtenidas en la escala ECS y las respuestas dadas al ítem correspondientes a la ideología política que se autoatribuían los sujetos. Sometidas las variables a un análisis de varianza, se obtuvo un valor de $\mathrm{F}=21,14 ; \mathrm{p}<0,001$, lo que indica que existe relación entre ambas. Una prueba de Scheffe señala que las diferencias entre las puntuaciones en la escala son significativas en todos los grupos establecidos por el ítem desde la derecha hasta la izquierda (ver Tabla 8) excepto entre los de izquierda y centro izquierda.

TABLA 8

Nivel de significación tras la prueba de Scheffe en las puntuaciones en la escala ECS según las ideologías políticas $(1=p<0,01 ; 5=p<0,05)$.

\begin{tabular}{|l|c|c|c|c|l|}
\hline & IZDA. & C-IZDA. & CENTRO & C-DCHA. & DCHA. \\
\hline IZDA. & & & & & \\
\hline C-IZDA. & & & & & \\
\hline CENTRO. & 5 & 1 & & & \\
\hline C-DCHA. & 1 & 5 & 1 & & \\
\hline DCIIA. & 1 & 1 & 5 & 5 & \\
\hline
\end{tabular}

En la escala todos los grupos tuvieron puntuaciones medias superiores a 48 , lo que indica que en todos ellos hay una actitud positiva hacia las cuestiones ambientales; no obstante, cuanto más próximo está un grupo a la izquierda más ambientalista es su puntuación, aunque dentro de los sujetos que participan, en alguna medida de esta ideología, la escala no discrimina. Esto hace pensar que el ambientalismo no es patrimonio de una ideología (izquierda-derecha), si bien los sujetos de izquierdas obtienen una puntuación más alta que los de derechas en la escala.

\section{B) Ambientalismo y energía nuclear}

Las actitudes ambientales y la energía nuclear han sido relacionadas en múltiples trabajos como se ha puesto de manifiesto en la introducción a este estudio empírico recurriendo a diversas estrategias. En la presente investigación la relación entre ambas variables ha sido estudiada a través del ítem comentado en el apartado anterior y la escala ECS.

Un análisis de la varianza entre los tres grupos definidos por el ítem y las puntuaciones obtenidas en la escala por los sujetos, dan lugar a un valor de $\mathrm{F}=32,06 ; \mathrm{p}<0,001$, lo que muestra que existe relación entre ambas variables. 


\section{6}

Un análisis posterior con la prueba de Scheffe pone de manifiesto que las diferencias en la escala entre los tres grupos son significativas, por lo que resulta que cuanto más opuesto es un grupo a la energía nuclear mayor puntuación alcanza en la escala de actitudes ambientales.

A la vista de los resultados cabría suponer que la situación consonante será estar en contra de la energía nuclear y ser proambientalista; o lo que es lo mismo, estar de acuerdo en que se pare la construcción de nuevas centrales nucleares e incluso eliminar las que funcionan en la actualidad y estar de acuerdo o bastante de acuerdo con la necesidad de leyes que protejan el medio ambiente a pesar de aumentar los precios y el paro. Sin embargo, cuando se cruzaron los valores de las variables actitud hacia la energía nuclear y proambientales, tal y como se acaban de definir, se encuentra una fuerte inconsistencia en 52 sujetos, el $7,2 \%$ de la muestra.

TABLA 9

Númeno de sujetos inconsistentes entre la respuesta dada a la energía nuclear y el ambientalismo.

\begin{tabular}{|l|c|c|c|}
\hline & MBDA & MBEDA & TOTAL \\
\hline NOCNUC & 68 & 41 & 109 \\
\hline SICNUC & 11 & 13 & 24 \\
\hline TOTAL & 79 & 54 & 133 \\
\hline
\end{tabular}

NOCNUC: En contra de las centrales nucleares.

SICNUC: A favor de las centrales nucleares.

MBDA: Muy o bastante de acuerdo en la escala.

MBEDA: Muy o bastante en desacuerdo en la escala.

Una inconsistencia mayor se observa en el trabajo de Webber (1982), un 35\% de la muestra que utiliza contestan de forma inconsistente a dos preguntas similares a las formuladas en este trabajo; muy probablemente, debido más al tipo de sujetos que a las coordenadas sociopolíticas de ambos contextos. En el caso de Webber, votantes californianos, y en el presente, estudiantes de Psicología.

No obstante, algunos comentarios merecen los 52 sujetos inconsistentes de la muestra, aunque en uno de los casos sea pequeño el número de los mismos. Los antinucleares, que no posponen el paro y la inflación ante los problemas ambientales, son de izquierdas significativamente; mientras que los que defienden el incremento de las centrales nucleares a favor del ambientalismo a pesar de generar paro e inflación, son de derechas. En ambos grupos, destacan los subgrupos de menores de 23 años y de mujeres.

Estos resultados vienen a poner en evidencia que la ideología está menos vinculada al ambientalismo que al desarrollo de la energía nuclear como fuente energética. No obstante, estos resultados junto con la incongruencia observada en los jóvenes y en las mujeres, deben prestarse atención en posteriores trabajos en donde se verifique si estos tipos de relación son extensibles a muestras más generales; y de ser así, intentar explicar la forma en que se suele resolver tal situación disonante. 
C) Ideología política y energía nuclear

La relación entre estas variables fue llevada a cabo a través de un cruce de las respuestas dadas al ítem correspondiente a la energía nuclear, con la ideología autoatribuida por los sujetos (Ver Tabla 10). Con el propósito de averiguar si existía relación entre las variables, se sometieron las frecuencias a un análisis $\chi^{2}$ donde se obtuvo un valor de 133,$76 ; \mathrm{p}<0,001$, lo que ponía de manifiesto su relación.

TABLA 10

Relación en porcentajes entre la ideología política autoatribuida y actitudes hacia la energía nuclear.

\begin{tabular}{|l|c|c|c|c|}
\hline & NO & NO MAS & SI MAS & TOTAL \\
& CENTRALES & CENTRALES & CENTRALES & \\
\hline IZDA. & 62,9 & 32,2 & 13,3 & 42,7 \\
C-IZDA. & 25,7 & 25,0 & 15,0 & 24,3 \\
CENTRO & 4,9 & 9,5 & 6,7 & 7,4 \\
C-DCHA. & 5,7 & 21,7 & 35,0 & 16,6 \\
DCHA. & 0,8 & 11,5 & 30,0 & 9,0 \\
\hline
\end{tabular}

A la vista de los resultados que aparecen en la Tabla 10, se puede afirmar que los sujetos de izquierdas mantienen posiciones más radicales frente a la energía nuclear que los de derechas. No obstante, los sujetos de la muestra en general se sitúan en posiciones en contra del crecimiento de esta fuente energética, ya que más del $63 \%$ de los sujetos de derechas tienen la opinión de que no se deben construir más centrales, aunque sigan funcionando las actuales.

\section{DISCUSION}

Los resultados obtenidos con la escala ECS permiten concluir que ésta discrimina entre proambientalistas y no proambientalistas, según el ítem patrón establecido al efecto. No obstante, dos aspectos quedan por resolver con respecto a la validez de la escala: por un lado, ver en qué medida un criterio externo confirma los resultados obtenidos en esta investigación, tal y como se apuntó en el apartado de resultados; y por otro, comprobar si la escala mide sólo el aspecto positivo de la relación del hombre con el medio ambiente. Esto último, puede resultar de interés, pues difícilmente pueden encontrarse personas que expresen verbalmente una actitud negativa hacia el medioambiente a pesar de realizar conductas que notoriamente son destructivas con respecto a él. Por consiguiente, parece interesante ver en qué medida las actitudes hacia el medio ambiente van a lo largo de un continuo proambiental versus antiambiental, o si ambos polos se refieren a conceptos distintos.

En otro orden de cosas, pero siempre dentro de los comentarios referidos a la escala de actitudes, ésta debería también ser sometida a un análisis de fiabilidad en un trabajo posterior.

Aunque en un primer momento puede objetarse la generalización de los resultados obtenidos dadas las características sociodemográficas de la muestra, ésta aporta también grandes ventajas al contemplar una fuerte homogeneidad en las 
variables: edad, nivel de educación y estatus socioeconómico. Por el contrario, la homogeneidad de la muestra, estudiantes de Psicología, favorece una mayor puntuación en la escala de actitudes proambientales, y por tanto la media de la muestra es muy superior al valor medio de la escala.

Los resultados obtenidos eran de esperar si se tiene en cuenta las características sociodemográficas de la muestra estudiada, como así señala la literatura sobre el tema. En este sentido, surge la necesidad de verificar empíricamente como funciona la escala, tanto con poblaciones extremas ante esta actitud como con la población española en general.

A pesar de los comentarios realizados sobre la escala ECS, hay que considerar que los resultados son satisfactorios, de tal modo que las propuestas que se acaban de señalar son consideraciones que podrían, por un lado, asegurar la utilidad de la escala; y por otro, profundizar en el concepto que subyace al significado de la actitud proambiental.

Por lo que respecta a las relaciones entre las tres variables actitudinales consideradas en esta investigación, merecen destacarse en este apartado dos comentarios de carácter diferente; uno sobre los aspectos metodológicos y otro, sobre alguna de las conclusiones derivadas de los resultados.

Por lo que respecta al primero, debe de mencionarse la necesidad de utilizar en trabajos posteriores, escalas de actitudes sobre la energía nuclear y la ideología política en un formato multiítem, de tal forma que los resultados obtenidos en esta investigación se pueden confirmar y matizar con pruebas estadísticas más potentes.

Por lo que se refiere a las relaciones entre las variables, merecen destacarse las siguientes observaciones: por un parte, el hecho de que los sujetos de izquierdas sean más proambientalistas que los de derechas, aunque todos puntúan con valores superiores al valor medio de la escala. Por consiguiente, el ambientalismo no es un patrimonio exclusivo de la izquierda como puede atribuirsè tesde ciertos medios. El discurso político de los sujetos que se dicen pertencientes a una ideología de izquierda o de derecha, se diferenciará en la intensidad de la preocupación por el ambiente, pero en ningún caso parece que responda a un contenido específico de una ideología.

Como viene considerando la bibliografía, hay una fuerte relación entre actitudes proambientales y actitudes hacia la energía nuclear, aunque esta última está maś vinculada a la ideología política que la anterior. En este trabajo, se muestran algunas incongruencias entre estas tres variables, lo que hace necesario profundizar en las relaciones entre ellas y en el caso de que muestren una estrecha vinculación, explicar las estrategias que utilizan las personas que sufren disonancia. 


\section{Referencias}

ArAgonés, J. I. (1985). La calidad ambiental: La conservación del entorno. En J. F. Morales Blanco, C. Huici, J. M. Fernández Dols (Comps.) Psicología Social Aplicada. Bilbao, Desclée de Brouwer.

Blas, F. A. y Aragonés, J. I. (1986). Conducta Ecológica Responsable: La conservación de la energía. En F. Jiménez Burillo y J. I. Aragonés (Comps.) Introducción a la Psicología Ambiental. Madrid, Alianza Editorial.

ButTel, F. H. y FLINN, W. L. (1978). The politics of Environmental concern: the Impacts of Party Identification and Political Ideology on Environmental Attitudes. Enviroment and Behavior, 10, 17-36.

Darley, J. y Gilbert, D. T. (1986). Social Psychological Aspects of Enviromental Psychology. En G. Lindzey y E. Aronson (Eds.) Handbook of Social Psychology. Nueva York, Randon House, $3 .^{\mathrm{a}}$ ed.

Dunlap, R. E. (1975). The impact of Political Orientation on Environmental Attitudes and Actions. Environment and Behavior, 7, 428-454.

Fischroff, B.; Lichtenstein, S.; Slovic, P.; Derby, S. L. y Keeney, R. L. (1981). Acceptable Risk. Cambridge. Cambridge University Press.

Geller, E. S. (1987). Applied Behavior Analysis and Environmental Psychology: From Strange Bedfellows to a Productive Marriage. En D. Stokols y I. Altman (Eds.) Handbook of Environmental Psychology. Nueva York, John Willey \& Sons.

Gifford, R. (1987). Enviromental Psichology. Principles and Practice. Boston, Allyn and Bacon, Inc.

Gray (Ed.) (1985). Ecological Beliefs and Bebaviors. Assessment and Change. Westport. Greenwood Pres.

Hensler, D. R. y Hesler, C. P. (1979). Evaluating Nuclear Power: Voter Choice on the California Nuclear Energy Initiative. Santa Mónica. Rand Corporation.

Levi, D. J. y Holder, E. E. (1986). Nuclear Power. The Dynamics of Acceptability. Environment and Behabior, 18, 385-395.

Maloney, S. F. y Ward, M. P. (1973). Ecology: let's hear from the people. American Psychologist, 30, 583-586.

Maloney, S. F.; Ward, M. P. y BRaucht, G. N. (1975). A revised scale for the measurement of ecological attitudes and knowledge. American Psychologist, 30, 787-790.

Milbrath, L. W. (1986). Environmental Beliefs and Values. En M. G.'Hermann (Ed.) Political Psychology. San Francisco. Jossey-Bass Publishers.

Otway, H. J. y Fisbhein, M. (1976). The Determinants of Attitude Formation: An application to Nuclear Power (Research Memorandum RM-76-80). Laxenburg, Austria: International Institute for Applied Systems Analysis.

Tognacci, L. N.; Weigel, R. H.; Wideen, M. F. y Vernon, D. T. (1972). Environmental Quality. How Universal is Public Concern? Environment and Behavior, 4, 73-86.

Van der Plig, J. (1985). Public Attitudes to Nuclear Energy: Salience and Anxiety. Journal of Environmental Psychology, 5, 87-97.

VAn Der Plig, J.; Eiser, R. J. y Spears, R. Attitudes toward Nuclear Energy. Familiarity and Salience. Environment and Behavior, 18, 75-93.

Van der Plig, J.; Van der Linden, J. y Ester, P. (1982). Attitudes to Nuclear Energy: Beliefs, Values and False Consensus. Joumal of Environmental Psychology, 2, 221-231.

VAN Liere, K. D. y DunLap, R. E. (1981). The social Bases of Environmental Concern: a Review of Hypotheses, Explanations, and Empirical Evidence. Public Opinion Quaterly, $44,181-197$.

Van Liere, K. D. y Dunlap, R. E. (1981). Environmental Concern. Does It Make a Difference How It's Measured? Environment and Behavior, 13, 651-676.

WEBBER, D. J. (1982). Is Nuclear Power just another Environmental Issue? An Analysis of California Voters. Environment and Behavior, 14, 72-86.

WEIGEL, R. H. (1977). Ideological and Demographic Correlates of Proecology Behavior. Journal of Social Psychology, 103, 39.47.

WeIGEL, R. y WeIGEL, J. (1978). Environmental Concern. The Delopment of Measure. Environment and Bebavior, 10, 3-15.

WoHLWILL, J. F. (1979). The social and Political Matrix Environmental Attitudes. An Analysis of Vote on the California Coastal Zone Regulation Act. Environment and Bebavior, $11,71-85$. 
APENDICE I

CUESTIONARIO DE ACTITUDES AMBIENTALES

UN GRUPO DE ESPECIALISTAS EN CIENCIAS AMBIENTALES PERTENECIENTES $A$ LA UNIVERSIDAD COMPLU.

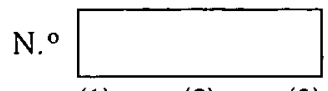

(1)

(2)

(3)

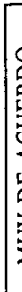

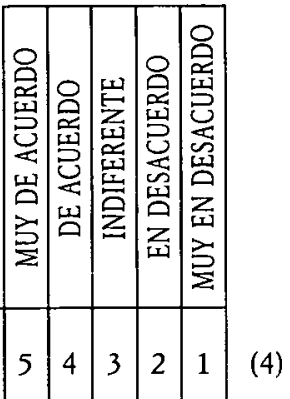

das para frenar la contaminación, ya que poca gente la regulará por sí misma.

2. No deberíamos preocuparnos por matar demasiados animales de caza porque a la larga las cosas se equilibrarán.

3. Estaría dispuesto a hacer sacrificios personales para reducir el ritmo de la contaminación aunque los resultados inmediatos no puedan parecer significativos.

4. La contaminación no afecta personalmente a mi vida.

5. Los beneficios de los productos de consumo modernos son más importantes que la contaminación que resulta de su producción y uso.

6. Debemos prevenir la extinción de cualquier tipo de animal, incluso si ello significa sacrificar algunas cosas para nosotros mismos.

7. En la escuela pública deberían ser impartidos cursos sobre la conservación de recursos naturales.

8. Aunque hay contaminación continua de lagos, ríos y aire, los procesos de purificación de la naturaleza pronto los retornan a lo normal.

9. Es muy improbable que la contaminación debida a la producción de energía llegue a ser excesiva, porque el gobierno tiene muy buenas inspecciones y agencias de control.

10. El gobierno debería suministrar a cada ciudadano una lista de agencias y organizaciones donde se pueda informar de los motivos de queja sobre la contaminación.

11. Los predadores tales como los halcones, cuervos, zorros y lobos que viven de las cosechas de grano y aves de corral de los granjeros deberían ser eliminados.

12. La actividad corriente de las organizaciones anticontaminación está realmente más interesada en romper con la sociedad que en luchar contra la contaminación.

13. Incluso si el transporte público fuera más eficiente de lo que es, yo prefiriría llevar mi coche al trabajo.

14. La industria está haciendo los mayores esfuerzos posibles para desarrollar tecnología anticontaminante efectiva.

15. Si pudiera daría tiempo, dinero o ambos para una organización como ADENA que trabaje para mejorar la calidad del ambiente.

16. Estaría dispuesto a aceptar un incremento de mis gastos de 5.000 ptas. el próximo año para promover el uso prudente de los recursos naturales. $\begin{array}{llllll}5 & 4 & 3 & 2 & 1\end{array}$

$\begin{array}{lllllll}5 & 4 & 3 & 2 & 1 & (13)\end{array}$

\begin{tabular}{|l|l|l|l|l}
5 & 4 & 3 & 2 & 1
\end{tabular}

$\begin{array}{lllllll}5 & 4 & 3 & 2 & 1 & (15\end{array}$

$\begin{array}{llllllll}5 & 4 & 3 & 2 & 1 & (16)\end{array}$

$\begin{array}{llllllll}5 & 4 & 3 & 2 & 1 & (17)\end{array}$

$\begin{array}{llllll}5 & 4 & 3 & 2 & 1\end{array}$

\begin{tabular}{l|l|l|l|l|l}
5 & 4 & 3 & 2 & 1 & $(19)$
\end{tabular} 